\title{
The role of pre-migration medical screening in high TB burden countries
}

IN THE FOURTH ARTICLE of the IJTLD State of the Art series on TB and migration in this issue of the Journal, Paul Douglas and colleagues introduce an interesting discussion around pre-migration medical screening programmes for migrants, and their potential role in capacity building and disease prevention in migrant-generating countries with a high prevalence of tuberculosis (TB). ${ }^{1}$ Collectively, over 2 million immigrants who are seeking visas for permanent entry to five low-incidence TB countries-Australia, Canada, New Zealand, the United Kingdom and the United States-pass through these pre-migration screening programmes every year, predominantly at sites in India, China, the Philippines and Viet Nam. The aim of these pre-migration screening programmes is to detect and treat active cases of TB (as well as detecting multi- and extensively drug-resistant TB), and thus support initiatives to reduce the burden of TB in migrant-receiving low-incidence TB countries, where in many settings TB rates remain high particularly in foreign-born nationals. ${ }^{2}$ Previously published data suggest pre-migration screening programmes are effective as a broad package of measures to ensure early diagnosis and treatment of migrants with TB. ${ }^{3}$ However, Douglas et al. conclude that premigration screening programmes may have additional positive impacts that stem from the collaborative efforts in high-burden TB countries, which include resourcing and increasing laboratory capacity (including drug susceptibility testing capacity) and drug availability, improving the infrastructure for direct observation of treatment, as well as building partnerships in high-burden countries to strengthen national TB programmes overall. The authors highlight that current pre-migration screening programmes potentially have additional benefits in terms of provision of health care worker training and tools for patient education, and that without these programmes many of these initiatives may not otherwise have been possible.

These programmes undoubtedly offer one more weapon in the armoury against the global threat of $\mathrm{TB}$ - the cornerstone of which is to seek out and treat active cases, which predominantly occur in low- and middle-income countries. However, as such predeparture programmes gain momentum and are expanded and resourced, robust research will be required to assess the true benefit of these initiatives among the wider population in high-burden, migrantsending countries, and to better understand the potential role of these programmes in influencing TB epidemiology in these contexts. As yet it is unclear to what extent these programmes are cost-effective and offer added value beyond merely resourcing the countries through their national TB control programmes which can be targeted to the specific incountry population requirements. Furthermore, questions remain around the long-term sustainability of pre-departure screening programmes.

Care, too, is needed to better understand whether pre-departure screening programmes are indeed migrant-friendly, and the extent to which they represent a barrier to individuals seeking to migrate. The practice of excluding migrants at borders based on health criteria is ominous: in Australia, a key reason for why visas have been refused in the past has been because migrants do not meet certain health criteria when they are screened pre-arrival. And it is only as recently as 2010 that the US government changed a policy spanning two decades that refused applications for short-term visas or lawful permanent residence to foreign-born nationals based on a positive diagnosis for the human immunodeficiency virus. Only time will tell which direction the new US administration will ultimately take on immigrant health screening.

The reality is that by far the majority of migrants from high-burden TB countries will bypass any kind of pre-departure screening programme altogether, and avoid or fail to be included in statutory immigrant screening programmes post-arrival. This will undoubtedly include undocumented migrants, some of whom will be at high risk for TB but who are afraid of approaching health services because of perceived or real links to immigration law enforcement. It is well understood, therefore, that multiple approaches to immigrant screening are needed in low-incidence settings, given that $\mathrm{TB}$ incidence rates remain high in migrants several years after relocation. ${ }^{4,5}$ Key to tackling TB in migrant-receiving, lowincidence TB countries is to ensure that migrants can access timely screening and health care should symptoms develop post-arrival. Going forward, this will involve tackling the considerable disparities in health care access faced by many migrants, including screening for $\mathrm{TB}$ and other infections, ${ }^{6,7}$ and the development of innovative strategies that take into account the unique social and cultural barriers faced by migrants to ensure uptake of screening and treatment completion for both latent and active TB. 
A more equitable approach to eliminating $\mathrm{TB}$ in countries such as the United States, Canada, Australia, New Zealand and the United Kingdom, therefore, will be to better resource and promote timely access to primary health care and screening for all at-risk migrant groups living there.

Sally Hargreaves* Raquel Duarte ${ }^{\dagger \neq \$}$ JON S. FRIEDLAND*

*International Health Unit, Imperial College London London, UK

†EpiUnit, Instituto de Saúde Pública, Universidade do Porto

*Faculdade de Medicina. Universidade do Porto

Porto

${ }^{\$}$ Centro Hospitalar de Vila Nova de Gaia/Espinho Vila Nova de Gaia Portugal e-mail: s.hargreaves@imperial.ac.uk

Conflicts of interest: none declared.

\section{References}

1 Douglas P, Posey D L, Zenner D, Robson J, Abubakar I, Giovinazzo G. Capacity strengthening through premigration TB screening programmes: IRHWG experiences. Int J Tuberc Lung Dis 2017; 21: 737-745.

2 European Centers for Disease Control and Prevention. Tuberculosis surveillance and monitoring in Europe. Stockholm, Sweden: ECDC, 2017.

3 Aldridge R W, Yates T A, Zenner D, White P J, Abubakar I, Hayward A C. Pre-entry screening programmes for tuberculosis in migrants to low-incidence countries: a systematic review and meta-analysis. Lancet Infect Dis 2014; 14: 1240-1249.

4 Lönnroth K, Migliori G B, Abubakar I, et al. Towards tuberculosis elimination: an action framework for low-incidence countries. Eur Respir J 2015: 45: 928-952.

5 Pareek M, Baussano I, Abubakar I, Dye C, Lalvani I. The impact of migration on tuberculosis epidemiology and control in highincome countries: a review. BMC 2016; 14: 48.

6 Seedat F, Hargreaves S, Friedland J S. Engaging new migrants in infectious disease screening: a qualitative semi-structured interview study of UK migrant community health-care leads. PLOS ONE 2014; 9(10): e108261

7 Médecins du Monde-Doctors of the World. International Network 2016 Observatory Report: access to health care for people facing multiple vulnerabilities in health in 31 cities in 12 countries. London, UK: Doctors of the World, Nov 2016. 